\title{
Minimally invasive treatment of infected pancreatic necrosis
}

\author{
Marek Wroński, Włodzimierz Cebulski, Maciej Słodkowski, Ireneusz W. Krasnodębski \\ Department of General, Gastroenterological and Oncological Surgery, Medical University of Warsaw, Warsaw, Poland
}

Prz Gastroenterol 2014; 9 (6): 317-324 DOI: $10.5114 /$ pg.2014.47893

Key words: acute pancreatitis, necrosectomy, minimally invasive treatment.

Address for correspondence: Marek Wroński MD, PhD, Department of General, Gastroenterological and Oncological Surgery, Medical University of Warsaw, 1 A Banacha St, 02-097 Warsaw, Poland, phone: +48 22599 24 82, e-mail: mwronski@vp.pl

\begin{abstract}
Infected pancreatic necrosis is a challenging complication that worsens prognosis in acute pancreatitis. For years, open necrosectomy has been the mainstay treatment option in infected pancreatic necrosis, although surgical debridement still results in high morbidity and mortality rates. Recently, many reports on minimally invasive treatment in infected pancreatic necrosis have been published. This paper presents a review of minimally invasive techniques and attempts to define their role in the management of infected pancreatic necrosis.
\end{abstract}

\section{Introduction}

Acute pancreatitis is usually mild and resolves without complications. In $10-20 \%$ of acute pancreatitis cases there is necrosis of the pancreas or extrapancreatic tissues [1]. Between $40 \%$ and $70 \%$ of patients with necrotising pancreatitis develop infected necrosis, which significantly worsens the prognosis [2]. The management of infected necrosis, irrespective of the technique used, is based on maximal removal of the necrotic tissues (necrosectomy) and drainage of the infected fluid. One of the most important factors determining the efficacy of necrosectomy is the timing of the operation. The optimal timing of surgical intervention in acute pancreatitis remains unclear. However, it is generally accepted that pancreatic necrosectomy should be performed as late as possible, and preferably after the fourth week of disease. Early necrosectomy is associated with high mortality and should be avoided. The only randomised trial that compared an early necrosectomy within $72 \mathrm{~h}$ from the beginning of acute pancreatitis with a late necrosectomy performed after 12 days of disease was terminated prematurely because of a high mortality rate in the former group (58\% vs. 27\%) [3]. Moreover, Besselink et al. [4] found that necrosectomy done at least 30 days since the onset of disease resulted in a low mortality rate of $8 \%$. In comparison, necrosectomy within the first 2 weeks of acute pancreatitis was associated with a mortality rate of $75 \%$, and which decreased to $45 \%$ if the operation was postponed until 2 weeks later.

The efficacy of necrosectomy is determined by the natural course of pancreatic necrosis and the degree of concomitant inflammation. The necrotic tissues become separated with time and undergo the process of liquefaction. Within the first 2-3 weeks of disease, pancreatic necrosis is poorly demarcated and it is difficult to discriminate between the necrotic and viable parenchyma. Attempts at necrosectomy in this phase of acute pancreatitis, at best, result in partial removal of the necrosis at the cost of severe bleeding and excessive resection of normal parenchyma. During the following weeks of disease, pancreatic necrosis becomes well separated and liquefies gradually. After the fourth week of acute pancreatitis, a solid-liquid collection is formed within a cavity covered by granulation tissue. Currently, this morphologic form of pancreatic necrosis is defined as walled-off necrosis [5], and necrosectomy at this stage of acute pancreatitis has the best chance of success.

Surgical necrosectomy through laparotomy is still the "gold standard" treatment of symptomatic pancreatic necrosis, despite its postoperative mortality rate of $20-40 \%$ and morbidity reaching as much as $78 \%[6,7]$. Traditional necrosectomy causes a "second hit", which results in worsening of organ dysfunction and paradox- 


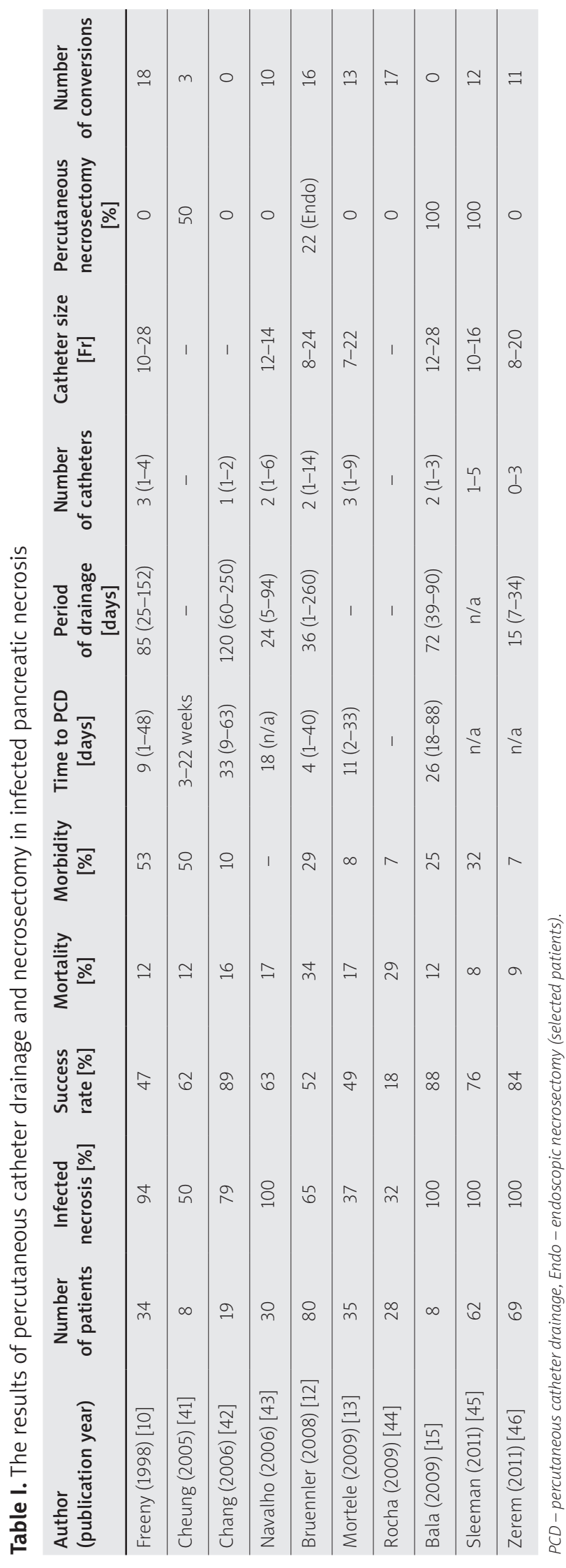

ically might transiently favour the progression of sepsis. This additional trauma might exceed the physiological reserves of the patient and lead to multiple organ failure, and death in some cases. Van Santvoort et al. [8] revealed that organ dysfunction is less common after minimally invasive necrosectomy, in comparison to open necrosectomy ( $12 \%$ vs. $40 \%, p=0.002)$. Similarly, Raraty et al. [9] observed lower APACHE II score and incidence of multiple organ failure in patients who underwent minimally invasive treatment of complicated acute pancreatitis.

Minimally invasive techniques have been introduced for the management of infected pancreatic necrosis in order to minimise the perioperative trauma and to limit its negative influence on organ function. Nowadays, endoscopic, radiological, laparoscopic, and hybrid techniques combining different minimally invasive modalities are used for the treatment of acute pancreatitis, depending on the experience of the institution.

\section{Percutaneous drainage and necrosectomy}

Percutaneous drainage is one of the first minimally invasive techniques employed for the treatment of infected pancreatic necrosis. Good results of percutaneous drainage in intra-abdominal abscesses encouraged its application also in patients with septic complications of acute pancreatitis. Freeny et al. [10] performed percutaneous drainage in 34 patients with infected pancreatic necrosis, with a success rate of $47 \%$. In this series, evacuation of the necrotic debris was facilitated by vigorous irrigation, use of multiple catheters, and their frequent upsizing. A median of three catheters and a median of four catheter exchanges were required per patient. Small-calliper catheters 10-14 Fr in diameter were used initially and thereafter exchanged for large-bore catheters 24$28 \mathrm{Fr}$ in size. Nevertheless, more than half of the patients, sooner or later, underwent surgical debridement.

The efficacy of percutaneous catheter drainage is varied and ranges between $18 \%$ and $89 \%$ (Table I). Horvath et al. [11] found that a decrease in size of the necrotic collection by $75 \%$ within 10-14 days predicted its success with $100 \%$ accuracy. However, such a rapid resolution of a necrotic collection might be expected only in case of the collections with a large proportion of liquefied contents. Percutaneous catheter drainage in infected pancreatic necrosis is usually regarded as a bridge treatment that allows the surgical intervention to be delayed. Despite this belief, percutaneous drainage proves to be the definitive treatment in many cases and there is no need for surgical intervention. The most important value of percutaneous drainage is ease of performance and the possibility of access into al- 
most any region of the abdominal cavity. Percutaneous drainage is usually performed under the guidance of computed tomography, which sometimes exposes the patients to a high dose of ionizing radiation. In a series reported by Bruennler et al. [12], a median of six contrast-enhanced computed tomography examinations were needed per patient (range: 1-23 exams).

The most important disadvantage of percutaneous drainage is the difficulty in establishing adequate outflow, due to frequent clogging of the catheters. Although large-bore catheters should provide better drainage of the particulate debris and promote resolution of the necrotic collections, Bruennler et al. [12] did not confirm the influence of the catheter size on the treatment results in acute pancreatitis. The most common complications of percutaneous catheter drainage are gastrointestinal fistulas, which occur with a rate of up to $20 \%[10,12]$. These fistulas are usually clinically not significant and do not require any specific treatment. The most serious complication of percutaneous drainage is haemorrhage due to local sepsis or incidental injury of a blood vessel. Two cases of a fatal haemorrhage from the splenic artery upon insertion of the catheter have been reported in the literature $[10,13]$.

To improve the efficacy of debridement, a technique of percutaneous necrosectomy was developed. It is based on mechanical fragmentation of the necrotic tissues and their extraction under the fluoroscopic guidance. This technique was introduced by Shonnard et al. [14], who removed the necrotic debris through the drain tract using the nitinol loops. Other authors used endoscopic baskets or tripod graspers for this purpose [15]. Percutaneous necrosectomy is more successful in the treatment of pancreatic necrosis than simple percutaneous drainage (Table I). The disadvantage of this technique is the lack of visual control during removal of the necrotic debris, which can result in incidental injury of the blood vessels or bowel wall, although this potential risk is not reflected in the literature.

\section{Minimally invasive retroperitoneal necrosectomy}

The advancement of percutaneous necrosectomy is minimally invasive retroperitoneal necrosectomy performed under the visual control provided by optical devices. This technique uses the retroperitoneal access through a mini-lumbotomy, usually left-sided, and there are two basic types of this technique. One type, called video-assisted retroperitoneal debridement (VARD), is in fact a hybrid technique combining open lumbotomy with a laparoscopic technique. This modality was introduced by Gambiez et al. [16], who originally removed the necrotic debris visualised by means of a medias- tinoscope through a small lumbotomy. This procedure can be performed without gas insufflation, and the initial debridement is often done blindly using open surgery instrumentation. A similar technique was used by van Santvoort et al. [17] and Castellanos et al. [18], but the visualisation of the necrotic cavity was provided by a laparoscope and a fibrescope, respectively.

Another type of minimally invasive necrosectomy is minimal access retroperitoneal pancreatic necrosectomy (MARPN), which was introduced by Carter et al. [19]. In this technique a nephroscope was originally used through a tract formed along a drain that was inserted during previous open necrosectomy. The visualisation of the necrosis was aided by instillation of saline, and the necrotic debris was removed through the working channel of the nephroscope. Later, this technique was also used for patients who had not been operated on, and the tract for placement of a nephroscope was created using percutaneous dilators. There are several modifications of this retroperitoneoscopic technique, and most of them require gas insufflation to achieve pneumoretroperitoneum. Horvath et al. [20] carried out retroperitoneal necrosectomy using two trocars, $10-12 \mathrm{~mm}$ in size, which were inserted through one small incision in the flank. In comparison, Bucher et al. [21] removed the necrotic debris using a 5-mm grasper and laparoscope, which were manipulated through a single trocar. One disadvantage of the aforementioned techniques is unstable pneumoretroperitoneum, which significantly impedes visualisation of the necrotic cavity and necrosectomy. These difficulties are avoided using the technique reported by Sileikis et al. [22], who inserted three trocars separately into the necrotic collection through the left lumbar region. Another modification requires a special SILS port (Covidien Polska) adopted for single incision laparoscopic surgery, which keeps a tight pneumoretroperitoneum and thus facilitates necrosectomy [23].

The success rate of minimally invasive necrosectomy is high and ranges from $60 \%$ to $100 \%$, whereas morbidity and mortality are relatively low (Table II). The principal reason for conversion to open surgery is lack of access to the necrotic collection or its part. In a series reported by Horvath et al. [11] 4 of 25 patients required surgical intervention due to a persistent collection at the base of the mesentery, although a retrocolic collection resolved. A further 6 patients in this series underwent open debridement because collection was not accessible through the left flank. In such patients, endoscopic necrosectomy might be a better option. Similarly to percutaneous catheter drainage, the main complications of video-assisted necrosectomy are gastrointestinal fistulas, especially pancreatic, and haemorrhage $[9,24]$. 


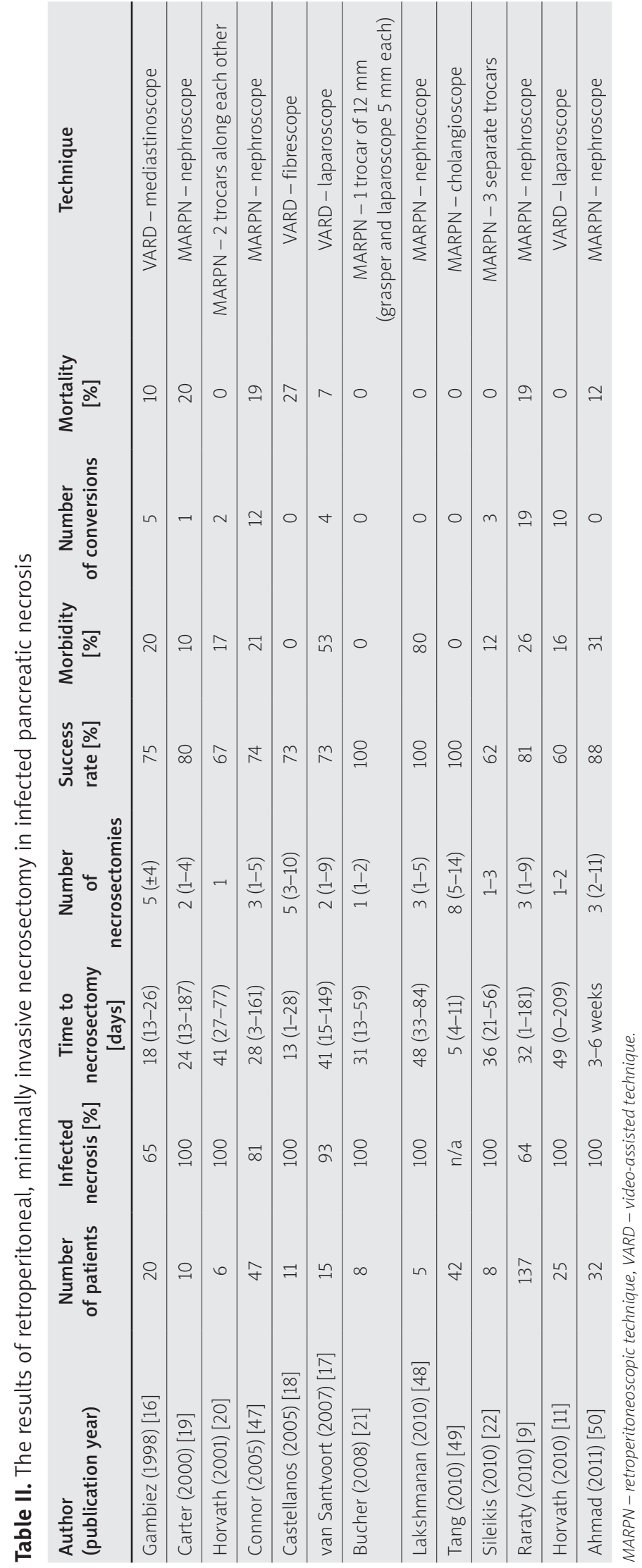

\section{Endoscopic necrosectomy}

Endoscopic drainage for the treatment of pancreatic necrosis was first performed by Baron et al. [25]. Their technique was similar to that employed in pancreatic cysts, but two stents $10 \mathrm{Fr}$ in size were inserted into the necrotic collection. Because of superinfection of the necrosis in the first patients, the following patients had a nasopancreatic catheter placed into the necrotic cavity for lavage and faster liquefaction of the necrotic debris. Nine of 11 patients recovered in this series.

Some modifications of endoscopic therapy have been suggested in order to improve evacuation of the necrotic debris. Varadarajulu et al. [26] used two or three access points through the stomach or duodenum. This technique was more successful than endoscopic drainage using only one access point (91.7\% vs. $52.1 \%$ ).

Further development of endoscopic therapy in infected pancreatic necrosis is endoscopic necrosectomy. Endoscopic necrosectomy was introduced by Seifert [27] and is currently one of the most common NOTES procedures (natural orifice transluminal endoscopic surgery). This technique relies on the removal of the necrosis through a $1.5-2 \mathrm{~cm}$ aperture that is created in the gastric or duodenal wall endoscopically. Endoscopic necrosectomy is best suited for patients with a walled-off necrosis located within $2 \mathrm{~cm}$ of the gastric or duodenal wall, and usually in the lesser sac. The first debridement session is often guided by endosonography, which facilitates the procedure and makes it safer, especially when there is no intraluminal impression on endoscopy.

The largest series of endoscopic necrosectomy was reported by a German group from the multicentre trial GEPARD (German Multicentre Study on Endoscopic Pancreatic Retroperitoneal Debridement) [28]. Ninety-three patients with acute pancreatitis were recruited into the trial. Endoscopic necrosectomy was successful in $80 \%$ of the patients. Overall mortality and morbidity were $7.5 \%$ and $26 \%$, respectively. The most common complications were bleeding and perforation into the free peritoneal cavity. There were two procedure-related fatalities in this series. One patient experienced a massive haemorrhage and died before a surgical intervention could be undertaken. Another patient suffered a fatal air embolism. Therefore, insufflation with carbon dioxide is recommended for endoscopic necrosectomy.

The efficacy of endoscopic necrosectomy is high and ranges between $75 \%$ and $100 \%$, whereas its mortality is relatively low, up to $15 \%$ (Table III). Notably, endoscopic necrosectomy is performed in a highly selected group of patients who usually have the necrotic collections limited to the lesser sac. Moreover, the interval between the onset of acute pancreatitis and endoscopic necrosectomy is longer (mean 45 days) in comparison 
to percutaneous drainage (mean 16 days), minimally invasive necrosectomy (mean 30 days), or open necrosectomy (mean 25 days).

The most common complication of endoscopic necrosectomy is bleeding into the postnecrotic cavity or, more often, from the gastric wall [28, 29]. A rare, but typical to this technique, complication is perforation into the peritoneal cavity resulting in diffuse peritonitis due to contamination with the purulent and necrotic material [28]. This latter complication requires urgent surgical treatment. The unquestionable advantage of endoscopic necrosectomy is a negligible rate of pancreatic fistulas.

Gardner et al. [30] from the Mayo Clinic carried out a trial comparing endoscopic necrosectomy with endoscopic drainage for the treatment of symptomatic pancreatic necrosis. Endoscopic necrosectomy had a significantly higher success rate than endoscopic drainage ( $88 \%$ vs. $45 \%)$, whereas morbidity and length of treatment were comparable. Gluck et al. [31] proposed a technique of dual modality drainage that combined endoscopic and percutaneous drainage. The development of this technique was inspired by the fact that some patients with necrotising pancreatitis recovered faster when they developed a duodenal fistula, which facilitated drainage of the necrotic debris. Compared with standard percutaneous drainage, the patients treated with dual drainage had shorter hospital stay (16 vs. 39 days), fewer CT examinations (7.5 vs. 13), fewer catheters (1 vs. 2), and shorter drainage time (65 vs. 136 days). None of the patients in the dual drainage group required open surgery, whereas 3 of 46 patients treated with percutaneous drainage alone underwent surgical intervention. The mortality rates were comparable in both groups ( $4 \%$ vs. 7\%). A similar technique was used by Becker et al. [32]. All seven patients in their series recovered by endoscopic drainage combined with percutaneous drainage. On the other hand, in a series reported by Papachristou et al. [33] 40\% of the patients who underwent endoscopic necrosectomy required additional percutaneous drainage due to a residual collection of infected necrosis that was inaccessible endoscopically. The authors concluded that the failure rate of endoscopic treatment is higher when the necrotic collection is above $15 \mathrm{~cm}$ in size or when it extends into the retrocolic region.

Recently Bakker et al. [34] published the results of a randomised trial PENGUIN (Pancreatitis, ENdoscopic transGastric versUs primary necrosectomy in patients with Infected Necrosis), which compared endoscopic necrosectomy with surgical debridement (VARD or open necrosectomy). Endoscopic necrosectomy showed lower postprocedural severity of proinflammatory response (measured by serum interleukin-6 level) and lower rate of major complications.

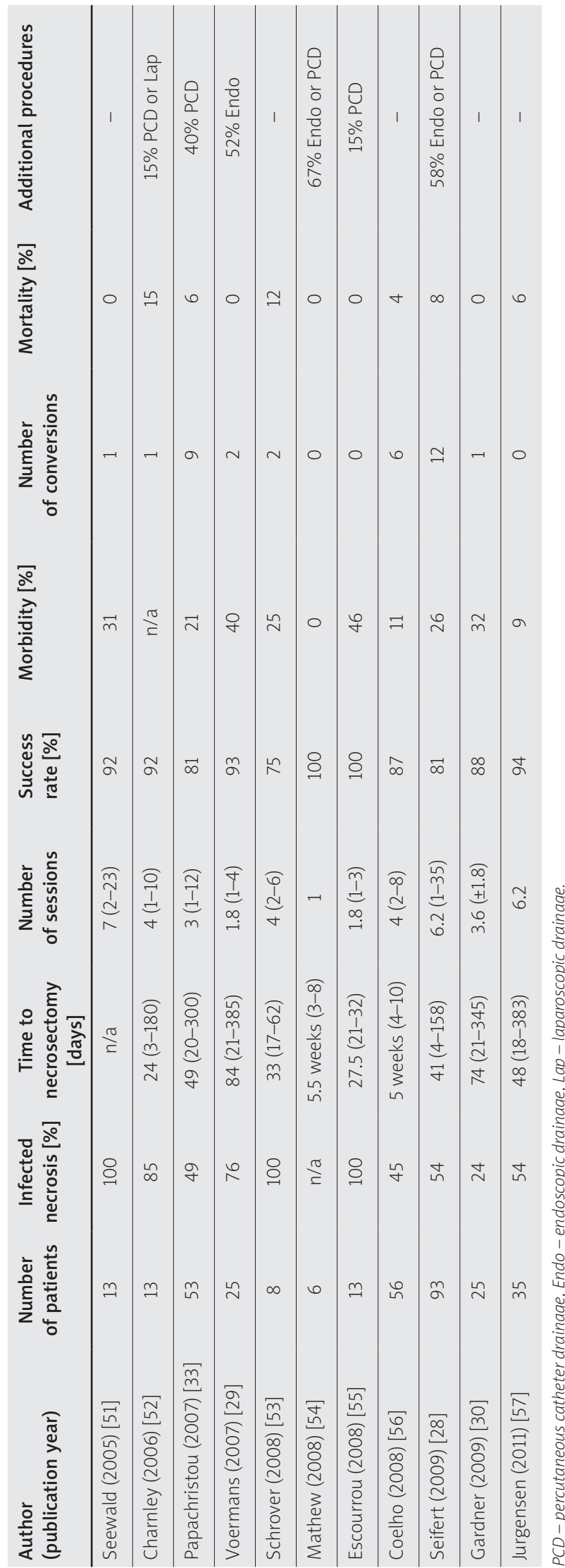

Przegląd Gastroenterologiczny 2014; 9 (6) 


\section{Laparoscopic necrosectomy}

Transperitoneal laparoscopic operations are rarely used for the treatment of acute pancreatitis due to technically difficult exposure of the pancreatic region and risk of contamination of the peritoneal cavity. Zhu et al. [35] performed laparoscopic operations in ten patients with severe acute pancreatitis, and nine of them recovered. The laparoscopic procedures were limited to lavage and drainage of the peripancreatic area. However, all the operations were performed within the first 24-72 h of the disease, which, in view of current management recommendations in acute pancreatitis, raises doubts about the rationale of these interventions. A similar laparoscopic technique and indications were reported in a series published by Zhou et al. [36]. Seven of 13 patients had laparoscopic drainage, whereas the remaining patients underwent retroperitoneoscopic necrosectomy. In comparison, Cuschieri et al. [37] carried out successful laparoscopic necrosectomy in 2 of 3 patients using a transperitoneal access through the mesentery of the transverse colon. One of their patients underwent open debridement. Parekh [38] used this transperitoneal transmesocolic access and a special device for hand-assisted surgery (Gelport) in 19 patients with acute pancreatitis. One patient in this series required conversion to open surgery because of bowel injury, and 2 other patients underwent open necrosectomy later.

Ammori [39] performed the first laparoscopic transperitoneal transgastric necrosectomy in a patient with infected pancreatic necrosis. The next four transgastric interventions were done in patients with sterile necrosis [40]. All the patients recovered, and there were no conversions to open surgery. The authors concluded that laparoscopic transgastric necrosectomy seems to be technically easier even than endoscopic necrosectomy.

\section{Selection of necrosectomy technique in the era of minimally invasive surgery}

Nowadays there are no clinical or radiological criteria allowing the prediction of which minimally invasive techniques might prove successful in patients with infected necrosis. Therefore, the choice of a minimally invasive procedure depends mainly on the expertise of the treating team and on the preferred technique used in the institution. There are many minimally invasive techniques available for the treatment of complicated acute pancreatitis. In some patients, it is useful to combine various techniques or to use them at different stages of the disease. On the other hand, open necrosectomy does not exclude the possibility of subsequent usage of minimally invasive procedures.
The principal problem common to all the minimally invasive techniques is the difficulty in removal of the necrotic debris and in the establishment of adequate drainage of the necrotic and purulent fluid. Therefore, it is often necessary to repeat sessions of necrosectomy and to use active lavage.

Pancreatic necrosis might become infected in the first week of acute pancreatitis, and the patient's condition sometimes does not allow for further delay in surgical intervention because of worsening multiple organ failure. In such situations, percutaneous drainage of the infected necrosis and peripancreatic collections might allow control of infection, stabilisation of the patient's condition, and postponement of the surgical intervention to a more convenient time.

Endoscopic treatment is used preferably in patients with the necrotic collections limited to the lesser sac, and in patients who require intervention at the later stages of disease when the necrosis is well liquefied. Retroperitoneal, minimally invasive necrosectomy seems to be an interesting technique for the treatment of patients with large necrotic collections extending down to the retrocolic regions, and also after adequate liquefaction of the necrosis. Although the results of minimally invasive techniques are encouraging, their use should not lead to a delay in appropriate treatment. Lack of clinical improvement should always be an indication for open necrosectomy, which is still the preferred choice in situations such as acute pancreatitis complicated by intra-abdominal compartment syndrome, colon ischaemia, and bowel perforation. On the other hand, percutaneous or endoscopic drainage might be life saving in patients who are critically ill and in whom general anaesthesia is contraindicated.

In conclusion, the role of minimally invasive techniques remains unclear; however, these techniques seem to be promising methods for the treatment of complicated acute pancreatitis. Minimally invasive techniques should be performed in specialised institutions with expertise in the management of severe pancreatitis. The selection of a minimally invasive procedure depends on the extent of necrosis, timing of intervention, patient's condition, and the experience and preference of the institution. In the near future, further developments in minimally invasive techniques and increasing popularity of hybrid methods for the treatment of infected pancreatic necrosis might be expected.

\section{References}

1. Uhl W, Warshaw A, Imrie C, et al. IAP Guidelines for the Surgical Management of Acute Pancreatitis. Pancreatology 2002; 2: 565-73.

2. Hartwig W, Werner J, Uhl W, et al. Management of infection in acute pancreatitis. J Hepatobiliary Pancreat Surg 2002; 9: 423-8. 
3. Mier J, Leon EL, Castillo A, et al. Early versus late necrosectomy in severe necrotizing pancreatitis. Am J Surg 1997; 173: 71-5.

4. Besselink MG, Verwer TJ, Schoenmaeckers EJ, et al. Timing of surgical intervention in necrotizing pancreatitis. Arch Surg 2007; 142: 1194-201.

5. Sheu Y, Furlan A, Almusa O, et al. The revised Atlanta classification for acute pancreatitis: a CT imaging guide for radiologists. Emerg Radiol 2012; 19: 237-43.

6. Rau B, Bothe A, Beger HG. Surgical treatment of necrotizing pancreatitis by necrosectomy and closed lavage: changing patient characteristics and outcome in a 19-year, single-center series. Surgery 2005; 138: 28-39.

7. Gotzinger P, Sautner T, Kriwanek S, et al. Surgical treatment for severe acute pancreatitis: extent and surgical control of necrosis determine outcome. World J Surg 2002; 26: 474-8.

8. van Santvoort HC, Besselink MG, Bakker OJ, et al. A step-up approach or open necrosectomy for necrotizing pancreatitis. N Engl J Med 2010; 362: 1491-502.

9. Raraty MG, Halloran CM, Dodd S, et al. Minimal access retroperitoneal pancreatic necrosectomy: improvement in morbidity and mortality with a less invasive approach. Ann Surg 2010; 251: 787-93.

10. Freeny PC, Hauptmann E, Althaus SJ, et al. Percutaneous CT-guided catheter drainage of infected acute necrotizing pancreatitis: techniques and results. AJR Am J Roentgenol 1998; 170: 969-75.

11. Horvath K, Freeny P, Escallon J, et al. Safety and efficacy of video-assisted retroperitoneal debridement for infected pancreatic collections: a multicenter, prospective, single-arm phase 2 study. Arch Surg 2010; 145: 817-25.

12. Bruennler T, Langgartner J, Lang S, et al. Outcome of patients with acute, necrotizing pancreatitis requiring drainage-does drainage size matter? World J Gastroenterol 2008; 14: 725-30.

13. Mortele KJ, Girshman J, Szejnfeld D, et al. CT-guided percutaneous catheter drainage of acute necrotizing pancreatitis: clinical experience and observations in patients with sterile and infected necrosis. AJR Am J Roentgenol 2009 192: 110-6.

14. Shonnard KM, McCarter DL, Lyon RD. Percutaneous debridement of infected pancreatic necrosis with nitinol snares. J Vasc Interv Radiol 1997; 8: 279-82.

15. Bala M, Almogy G, Klimov A, et al. Percutaneous "stepped” drainage technique for infected pancreatic necrosis. Surg Laparosc Endosc Percutan Tech 2009; 19: e113-8.

16. Gambiez LP, Denimal FA, Porte HL, et al. Retroperitoneal approach and endoscopic management of peripancreatic necrosis collections. Arch Surg 1998; 133: 66-72.

17. van Santvoort HC, Besselink MG, Bollen TL, et al. Case-matched comparison of the retroperitoneal approach with laparotomy for necrotizing pancreatitis. World J Surg 2007; 31: 1635-42.

18. Castellanos G, Pinero A, Serrano A, et al. Translumbar retroperitoneal endoscopy: an alternative in the follow-up and management of drained infected pancreatic necrosis. Arch Surg 2005; 140: 952-5.

19. Carter CR, McKay CJ, Imrie CW. Percutaneous necrosectomy and sinus tract endoscopy in the management of infected pancreatic necrosis: an initial experience. Ann Surg 2000; 232: 175-80.
20. Horvath KD, Kao LS, Ali A, et al. Laparoscopic assisted percutaneous drainage of infected pancreatic necrosis. Surg Endosc 2001; 15: 677-82.

21. Bucher P, Pugin F, Morel P. Minimally invasive necrosectomy for infected necrotizing pancreatitis. Pancreas 2008; 36: 113-9.

22. Sileikis A, Beisa V, Simutis G, et al. Three-port retroperitoneoscopic necrosectomy in management of acute necrotic pancreatitis. Medicina (Kaunas) 2010; 46: 176-9.

23. Wronski M, Cebulski W, Slodkowski M, et al. Retroperitoneal minimally invasive pancreatic necrosectomy using single-port access. Surg Laparosc Endosc Percutan Tech 2012; 22: e8-11.

24. Connor S, Alexakis N, Raraty MG, et al. Early and late complications after pancreatic necrosectomy. Surgery 2005; 137 : 499-505.

25. Baron TH, Thaggard WG, Morgan DE, et al. Endoscopic therapy for organized pancreatic necrosis. Gastroenterology 1996; 111: 755-64.

26. Varadarajulu S, Phadnis MA, Christein JD, et al. Multiple transluminal gateway technique for EUS-guided drainage of symptomatic walled-off pancreatic necrosis. Gastrointest Endosc 2011; 74: 74-80.

27. Seifert H, Wehrmann T, Schmitt T, et al. Retroperitoneal endoscopic debridement for infected peripancreatic necrosis. Lancet 2000; 356: 653-5.

28. Seifert H, Biermer M, Schmitt W, et al. Transluminal endoscopic necrosectomy after acute pancreatitis: a multicentre study with long-term follow-up (the GEPARD Study). Gut 2009; 58: 1260-6.

29. Voermans RP, Veldkamp MC, Rauws EA, et al. Endoscopic transmural debridement of symptomatic organized pancreatic necrosis (with videos). Gastrointest Endosc 2007; 66: 909-16.

30. Gardner TB, Chahal P, Papachristou GI, et al. A comparison of direct endoscopic necrosectomy with transmural endoscopic drainage for the treatment of walled-off pancreatic necrosis. Gastrointest Endosc 2009; 69: 1085-94.

31. Gluck M, Ross A, Irani S, et al. Dual modality drainage for symptomatic walled-off pancreatic necrosis reduces length of hospitalization, radiological procedures, and number of endoscopies compared to standard percutaneous drainage. J Gastrointest Surg 2012; 16: 248-56.

32. Becker V, Huber W, Meining A, et al. Infected necrosis in severe pancreatitis: combined nonsurgical multi-drainage with directed transabdominal high-volume lavage in critically ill patients. Pancreatology 2009; 9: 280-6.

33. Papachristou GI, Takahashi N, Chahal P, et al. Peroral endoscopic drainage/debridement of walled-off pancreatic necrosis. Ann Surg 2007; 245: 943-51.

34. Bakker OJ, van Santvoort HC, van Brunschot S, et al. Endoscopic transgastric vs surgical necrosectomy for infected necrotizing pancreatitis: a randomized trial. JAMA 2012; 307: 1053-61.

35. Zhu JF, Fan XH, Zhang XH. Laparoscopic treatment of severe acute pancreatitis. Surg Endosc 2001; 15: 146-8.

36. Zhou ZG, Zheng YC, Shu Y, et al. Laparoscopic management of severe acute pancreatitis. Pancreas 2003; 27: e46-50.

37. Cuschieri SA, Jakimowicz JJ, Stultiens G. Laparoscopic infracolic approach for complications of acute pancreatitis. Semin Laparosc Surg 1998; 5: 189-94.

38. Parekh D. Laparoscopic-assisted pancreatic necrosectomy: a new surgical option for treatment of severe necrotizing pancreatitis. Arch Surg 2006; 141: 895-902. 
39. Ammori BJ. Laparoscopic transgastric pancreatic necrosectomy for infected pancreatic necrosis. Surg Endosc 2002; 16: 1362.

40. Owera AM, Ammori BJ. Laparoscopic endogastric and transgastric cystgastrostomy and pancreatic necrosectomy. Hepatogastroenterology 2008; 55: 262-5.

41. Cheung MT, Ho CN, Siu KW, et al. Percutaneous drainage and necrosectomy in the management of pancreatic necrosis. ANZ J Surg 2005; 75: 204-7.

42. Chang YC, Tsai HM, Lin XZ, et al. No debridement is necessary for symptomatic or infected acute necrotizing pancreatitis: delayed, mini-retroperitoneal drainage for acute necrotizing pancreatitis without debridement and irrigation. Dig Dis Sci 2006; 51: 1388-95.

43. Navalho M, Pires F, Duarte A, et al. Percutaneous drainage of infected pancreatic fluid collections in critically ill patients: correlation with C-reactive protein values. Clin Imaging 2006; 30: 114-9.

44. Rocha FG, Benoit E, Zinner MJ, et al. Impact of radiologic intervention on mortality in necrotizing pancreatitis: the role of organ failure. Arch Surg 2009; 144: 261-5.

45. Sleeman D, Levi DM, Cheung MC, et al. Percutaneous lavage as primary treatment for infected pancreatic necrosis. J Am Coll Surg 2011; 212: 748-52.

46. Zerem E, Imamovic G, Susic A, et al. Step-up approach to infected necrotising pancreatitis: a 20-year experience of percutaneous drainage in a single centre. Dig Liver Dis 2011; 43 478-83.

47. Connor S, Raraty MG, Howes N, et al. Surgery in the treatment of acute pancreatitis - minimal access pancreatic necrosectomy. Scand J Surg 2005; 94: 135-42.

48. Lakshmanan R, Iyer SG, Lee VT, et al. Minimally invasive retroperitoneal pancreatic necrosectomy in the management of infected pancreatitis. Surg Laparosc Endosc Percutan Tech 2010; 20: e11-5.

49. Tang LJ, Wang T, Cui JF, et al. Percutaneous catheter drainage in combination with choledochoscope-guided debridement in treatment of peripancreatic infection. World J Gastroenterol 2010; 16: 513-7.

50. Ahmad HA, Samarasam I, Hamdorf JM. Minimally invasive retroperitoneal pancreatic necrosectomy. Pancreatology 2011 11: $52-6$

51. Seewald S, Groth S, Omar S, et al. Aggressive endoscopic therapy for pancreatic necrosis and pancreatic abscess: a new safe and effective treatment algorithm (videos). Gastrointest Endosc 2005; 62: 92-100.

52. Charnley RM, Lochan R, Gray H, et al. Endoscopic necrosectomy as primary therapy in the management of infected pancreatic necrosis. Endoscopy 2006; 38: 925-8.

53. Schrover IM, Weusten BL, Besselink MG, et al. EUS-guided endoscopic transgastric necrosectomy in patients with infected necrosis in acute pancreatitis. Pancreatology 2008; 8: 271-6.

54. Mathew A, Biswas A, Meitz KP. Endoscopic necrosectomy as primary treatment for infected peripancreatic fluid collections (with video). Gastrointest Endosc 2008; 68: 776-82.

55. Escourrou J, Shehab H, Buscail L, et al. Peroral transgastric/ transduodenal necrosectomy: success in the treatment of infected pancreatic necrosis. Ann Surg 2008; 248: 1074-80.
56. Coelho D, Ardengh JC, Eulalio JM, et al. Management of infected and sterile pancreatic necrosis by programmed endoscopic necrosectomy. Dig Dis 2008; 26: 364-9.

57. Jurgensen C, Neser F, Boese-Landgraf J, et al. Endoscopic ultrasound-guided endoscopic necrosectomy of the pancreas: is irrigation necessary? Surg Endosc 2011; 26: 1359-63.

Received: 21.07.2012

Accepted: 15.11.2012 